\title{
The Management of Staphylococcal Toxic Shock Syndrome. A Case Report
}

\author{
Victoria Bîrluțiu1,2*, Ofelia Criștiu³, Marius Baicu4, Rareș Mircea Bîrluțiu1,5 \\ 1 Lucian Blaga University of Sibiu, Faculty of Medicine, Sibiu, Romania \\ 2 Sibiu County Clinical Emergency Hospital, Infectious Diseases Department, Sibiu, Romania \\ 3 Pediatric Clinical Hospital Sibiu, Infectious Diseases Department, Sibiu, Romania \\ 4 Pediatric Clinical Hospital Sibiu, Intensive Care Department, Sibiu, Romania \\ 5 Foişor Clinical Hospital of Orthopedics, Traumatology and Osteoarticular Tuberculosis, Bucharest, Romania
}

\begin{abstract}
Staphylococcal toxic shock syndrome (TSS) is most frequently produced by TSS toxin-1 (TSST-1) and Staphylococcal enterotoxin B (SEB), and only rarely by enterotoxins A, C, D, E, and $\mathrm{H}$. Various clinical pictures can occur depending on severity, patient age and immune status of the host. Severe forms, complicated by sepsis, are associated with a death rate of 50-60\%. The case of a Caucasian female infant, aged seven weeks, hospitalized with a diffuse skin rash, characterized as allergodermia, who initially developed TSS with axillary intertrigo, is reported.

TSS was confirmed according to 2011 CDC criteria, and blood cultures positive for Methicillin-sensitive Staphylococcus aureus (MSSA). Severe development occurred initial, including acidosis, consumption coagulopathy, multiple organ failures (MOF), including impaired liver and kidney function. Central nervous system damage was manifest by seizures. Clinical management included medical supervision by a multidisciplinary team of infectious diseases specialist and intensive care specialist, as well as the initiation of a complex treatment plan to correct hydro electrolytic imbalances and acidosis. This treatment included antibiotic and antifungal therapy, diuretic therapy, immunoglobulins, and local treatment similar to a patient with burns to prevent superinfection of skin and mucous membranes lesions. There was a favourable response to the treatment with resolution of the illness.
\end{abstract}

Keywords: Staphylococcal toxic shock syndrome, TSS, management

Received: 02 February 2016 / Accepted: 05 April 2016

\section{INTRODUCTION}

Staphylococcal toxic shock syndrome (TSS) presents as various clinical pictures dependant on severity, age and immune status of the host and is associated with a death rate of $50-60 \%$ in severe forms complicated by sepsis. When Staphylococcus aureus is the causative bacteria, TSS is caused by staphylococcus toxin, especially TSS toxin-1 (TSST-1), staphylococcal enterotoxin $\mathrm{B}$ and rarely to enterotoxins types $\mathrm{A}, \mathrm{C}, \mathrm{D}$, $\mathrm{E}$ and $\mathrm{H}$. These act as super antigens, activating the $\mathrm{T}$ lymphocytes with massive release of proinflammatory cytokines responsible for the following clinical picture of fever, rash, septic shock, and multiple organ failures [1-4]. Staphylococcal enterotoxins and TSST-1 bind to the major histocompatibility complex (MHC) class II molecules, activating $\mathrm{V} \beta 2+\mathrm{T}$ cells, which account for
$10 \%$ of the total number of $\mathrm{T}$ lymphocytes $[5,6]$ and which express CD45RO as evidence of lymphocyte activation.

Epidemiological studies conducted in the UK and Ireland reported an incidence rate for TSS of 0.38 per 100000 children [7]. Both children and adults cases of varying severity and death rates of up to $50-60 \%$ in septic shock forms, are described in the literature [8-10].

According to CDC criteria [11], TSS diagnosis is supported by the following clinical and laboratory criteria:

- body temperature $>38.9^{\circ} \mathrm{C}\left(102.02^{\circ} \mathrm{F}\right)$

- systolic blood pressure $<90 \mathrm{mmHg}$

- presence of a disseminated rash

- scaly skin, 1-2 weeks after onset, localized especially in the palmar-plantar level

\footnotetext{
Correspondence to: Victoria Bîrluțiu, Address: Lucian Blaga Str No. 2A, Sibiu, 550169, Romania, Mobile: 0040744875751. E-mail address: victoriabirlutiu@yahoo.com

Ofelia Criștiu: Pediatric Clinical Hospital Sibiu, Infectious Diseases Department, Transilvaniei Str. No. 2-4 Sibiu, Romania

Marius Baicu: Pediatric Clinical Hospital Sibiu, Intensive Care Department, Str. Gheorghe Barițiu Str. No. 1-3, Sibiu, Romania

Rareș Mircea Bîluțị: Foișor Clinical Hospital of Orthopedics, Traumatology and Osteoarticular Tuberculosis, Ferdinand I Bvd No. 35-37, Bucharest, Romania
} 
- multiple organ damage associated with at least three of the following:

- gastrointestinal tract, manifested by vomiting

- diarrhoea

- severe muscle damage, highlighted by increasing creatine phosphokinase (CPK)

- mucosal damage, oral, conjunctival or genital

- renal, hepatic functions failure, thrombocytopenia (platelet count $<100,000 / \mathrm{mm}^{3}$ )

- central nervous system damage, manifested by confusion with or without focal neurologic signs.

- identification of Staphylococcus aureus

- exclusion of other aetiologies from cerebrospinal fluid(CSF) studies or blood cultures i.e. other bacterial infections such as streptococcal infections, Rickettsia, leptospirosis and viral infections.

\section{DCASE REPORT}

The case is presented of a seven week-old female Caucasian infant, who was hospitalized in the Paediatric Department for a generalized skin rash, initially thought to be allergodermia. Initially, treatment with antihistamines and corticosteroids was started. During the following four days of hospitalization, persistence of the rash was recorded, accompanied by the occurrence of fever and diarrhoea. The infant was referred to the Infectious Diseases Department (IDD), after a positive rapid diagnostic test detected rotavirus antigen. Before this episode, the baby showed no other adverse conditions. She was a second full-term baby, delivered naturally in cranial presentation, a birth weight of 2820g, Apgar score 10, initially breastfed for one week and subsequently with cow's milk. The baby had not received medication for prevention of rickets.

At the time of referral to the IDD the infant presented with a weight of $4300 \mathrm{~g}$, fever $\left(39.7^{\circ} \mathrm{C}\right)$, malaise, generalized rash, tendency of scaling in flaps, particularly at the palmar-plantar level, bilaterally axillary and cervical intertrigo, eyelid and ankle oedema, vesicular breath sounds, respiratory rate of 40/min, $\mathrm{SaO} 296 \%$, heart rate 160-190/min, blood pressure 75/30 $\mathrm{mmHg}$, fried lips, raspberry exfoliated tongue, abdominal distension, hepato-splenomegaly, rapid intestinal transit, refusal of food, oliguria, horizontal nystagmus, tendency to opisthotonus, limb spasticity, presence of deep tendon reflexes, and right hemibody myoclonus.

A lumbar puncture was performed showinga hypertensive cerebrospinal fluid (CSF) with no cytological or biochemical changes. Laboratory examinations showed the following: creatinine $1.73 \mathrm{mg} / \mathrm{dl}$, BUN (blood urea nitrogen) in dynamics 64.4-83.8-98.7-107.2-25.6 mg/ $\mathrm{dL}$, glucose 40-41-33-65-67-48-87mg/dl, $\mathrm{pH}=7.258$, lactate $=53-107-70 \mathrm{mg} / \mathrm{dl}$, base excess (cBaseECF) -8.8; -16.8-14.5 $\mathrm{mmol} / \mathrm{L}, \mathrm{C}$-reactive protein (CRP): 6-212-194-164-99 -52 mg/dL, fibrinogen 80.9-19 mg/ dl 149.9-216.7 (180-380 mg/dl), procalcitonin PCT 10 $\mathrm{ng} / \mathrm{ml}$, total protein (TP) 3.68-6.08 mg/dl, TSH $0.46 \mathrm{U} /$ $\mathrm{ml}$, FT4 $12.67 \mathrm{pmol} / \mathrm{L}$, FT3 $1.24 \mathrm{pmol} / \mathrm{L}$, stool and nasal swab positive for Candida +++ and blood cultures positive for Staphylococcus aureus, sensitive to chloramphenicol, ciprofloxacin, levofloxacin, moxifloxacin, ofloxacin, oxacillin, trimethoprim/sulfamethoxazole, clindamycin, erythromycin, gentamicin, resistant to penicillin.

Other examinations showed: total bilirubin $(\mathrm{TB})=$ $5.12-3.44 \mathrm{mg} / \mathrm{dL}$, direct bilirubin $(\mathrm{DB})=3.79-2.28 \mathrm{mg} /$ $\mathrm{dl}$, aspartate aminotransferase (AST) 111-85 U/L, alanine aminotransferase (ALT) 56-75 U/L, prothrombin time PT (Quick time) 21.7-17.8-19.6 -13.7-11.8 s (N 13-17) prothrombin activity 38.2-47.8-60 -81.6-95\%, INR 1.90-1.57-1.3-1.12-1.03 (0.82-1.25), APTT 82.8 sec. -58.2 - 34.1-36.7 (24.8-37.5), and fibrin monomers $(++++)$.

A complete blood count ( $\mathrm{CBC}$ ) showed the following changes: white blood cells (WBC) 9.08-14.17-43.40-

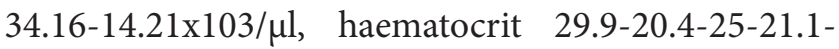
20.1\%, haemoglobin 10.9-7.3-7.6-8-6.4g/dl, platelets

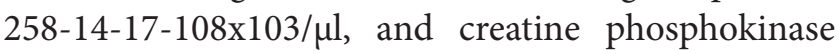
(CPK) $2384 \mathrm{mg} / \mathrm{dl}$ (N: 26-192 mg/dl).

Cranial ultrasonography revealed no pathological changes. Abdominal ultrasound was also normal, except for a plied gallbladder, abdominal distension, with the fluctuation of air and semi-fluid content leakage.

The following treatment was initiated: correction of the hydro-electrolytic and acid-base imbalances, inotropic support, administration of red cell mass (RCM), platelet concentrate, fresh frozen plasma (FFP), antibiotic treatment with meropenem, teicoplanin and antibiotic de-escalation to oxacillin after skin wound healing, fluconazole, intravenous immunoglobulin $30 \mathrm{ml} /$ day, albumin, forced diuresis with mannitol and furosemide $3 \times 2 \mathrm{mg} /$ day, proton-pump inhibitors (PPIs), probiotics, anticonvulsants - levetiracetam (Keppra ${ }^{\circledR}$ ) $2 \times 0.4 \mathrm{ml}$, and local skin therapy. The results of the treatment were defervescence, healing of skin and mucous lesions, resumption of diuresis $(150 \mathrm{ml} /$ day $)$, correction 
of renal and hepatic functions and normalization of the inflammatory markers.

The baby was discharged from the hospital three weeks after admission. The parents were recommended to implement hygiene measures, administer age appropriate artificial nutrition and initiate rickets prevention and vitamin D deficiency measures.

\section{DISCUSSIONS}

Staphylococcal TSS is considered in the UK, to be a condition specific to older children, compared to streptococcal toxic shock [7]. In the US, alterations in the fabrication and usage of tampons led to a decrease in staphylococcal TSS, although the incidence of nonmenstrual staphylococcal TSS increased. Nonmenstrual TSS and menstrual TSS occur with equal incidence [12]. In contrast, since 1992 in Japan, the occurrence of TSS cases in new-borns (neonatal TSS-like exanthematous disease (NTED)) has coincided with the emergence of strains of methicillin-resistant Staphylococcus aureus producing TSST-1, which is frequently associated with thrombocytopenia. NTED is explained by the vnew-born's immune tolerance to TSST-1, T cells suppression, the release of large amounts of anti-inflammatory cytokines, like interleukin IL-10 in concentrations $>1200 \mathrm{pg} / \mathrm{mL}$ [13], responsible for the presence of anergy, the immune response to superantigens being age-dependent [14].

The present case is confirmed by taking into account all six criteria of the CDC definition. The commencement of the condition was characterised by the intertrigo lesions, the isolation of Staphylococcus from blood cultures, the presence of skin rash (Figures 1,2), mucosal lesions (Figure 3), palmar-plantar scaling (Figure 4), renal and hepatic damage, and central nervous system impairment associated with myositis.

Although digestive events can be interpreted in the context of rotavirus co-infection, the baby presented with acute liver and renal failure, severe myositis, with CPK values 12 times higher than normal, consumption coagulopathy, severe thrombocytopenia, severe metabolic acidosis and severe damage to the central nervous system, right hemibody myoclonus, horizontal nystagmus, and spasticity. Severe hypoglycaemia was also recorded, which resolved following clinical and biological improvement, and the elimination of changes suggestive of consumption coagulopathy.

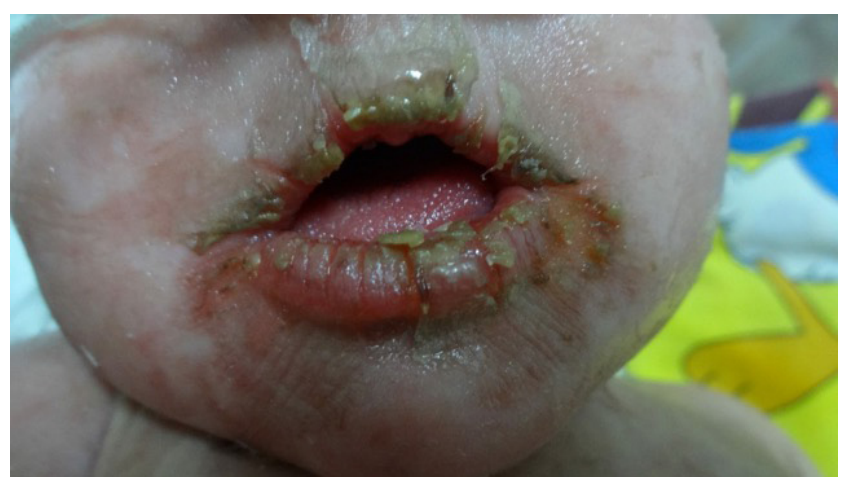

Fig. 1. Skin facial lesions in a case with Staphylococcal Toxic Shock Syndrome

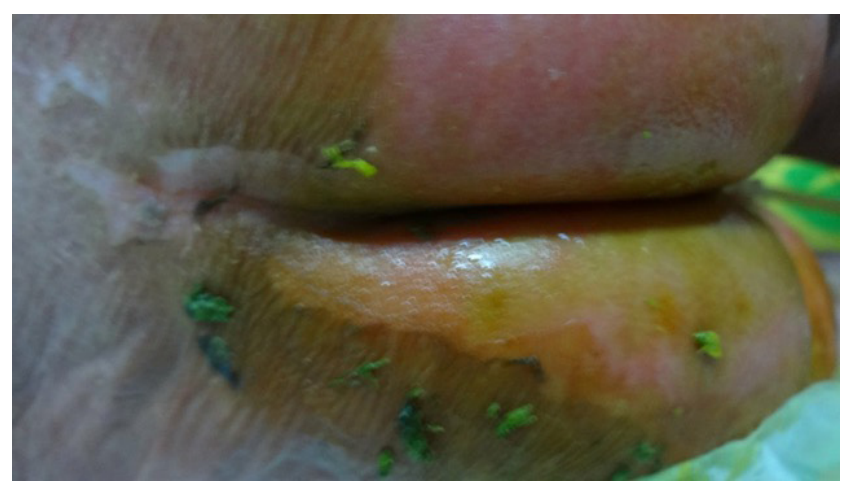

Fig. 2. Skin rash in a case with Staphylococcal Toxic Shock Syndrome

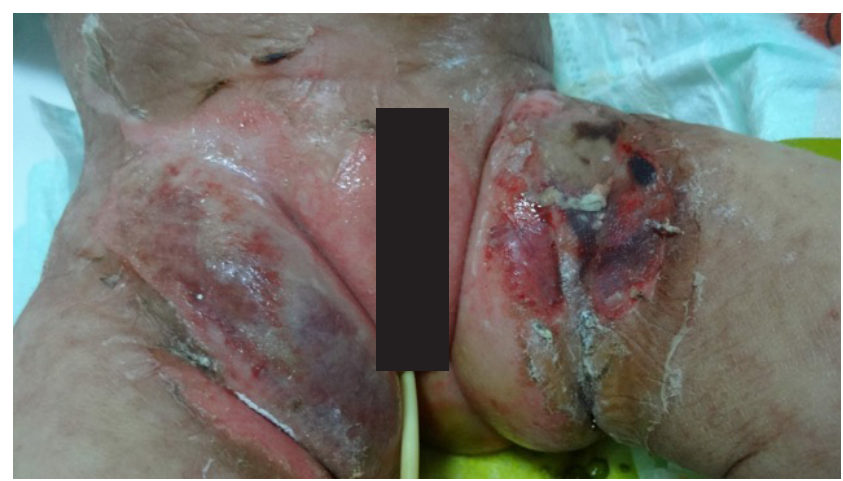

Fig. 3. Mucosal lesions in a case with Staphylococcal Toxic Shock Syndrome

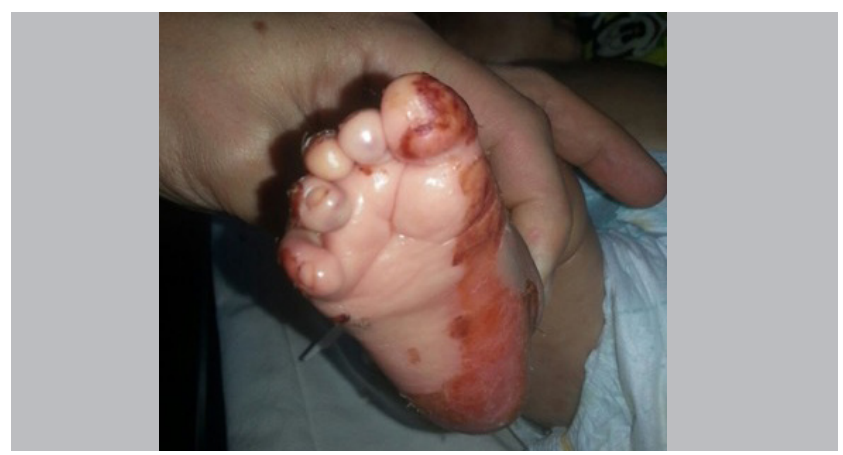

Fig. 4. Plantar scaling in a case with Staphylococcal Toxic Shock Syndrome 
Treatment and monitoring of the baby were undertaken by a multidisciplinary team in the ICU. This included the use of isolation and incubator conditions and maintaining aseptic measures throughout. This resulted in the favourable resolution of the condition without complications or superinfection of skin areas or damaged mucous membranes.

\section{CONCLUSION}

Although TSS is considered rare in infants, occurrence of fever and exanthema, regardless of patient age, should point to the possible differential diagnosis of staphylococcal or streptococcal toxic shock.

Recognition of TSS diagnosis is paramount in the proper management of the disease to improve the survival rate of patients.

\section{- CONSENT SECTION}

Written informed consent was obtained for publication of this case report and any accompanying images from the patient's parents. The study was accepted by the Ethics Committee of the hospital.

\section{DONFLICT OF INTEREST:}

Nothing to declare

\section{DEFERENCES}

1. Silversides JA, Lappin E, Ferguson AJ. Staphylococcal Toxic Shock Syndrome: Mechanisms and Management. Curr Infect Dis Rep. 2010;12:392-400.

2. Amagai M, Yamaguchi T, Hanakawa $Y$, Nishifuji K, Sugai M, Stanley JR. Staphylococcal exfoliative toxin B specifically cleaves desmoglein. J Invest Dermatol. 2002;118:845-50.

3. Hanakawa Y, Stanley JR. Mechanisms of blister formation by staphylococcal toxins. J Biochem. 2004;136:747-50.

4. Ladhani S. Understanding the mechanism of action of the exfoliative toxins of Staphylococcus aureus. FEMS Immunol Med Microbiol. 2003;39:181-9.

5. Uchiyama T, Yan X-J, Imanishi K, Yagi J. Bacterial superantigensmechanism of $T$ cell activation by the superantigens and their role in the pathogenesis of infectious diseases. Microbiol Immunol. 1994;38:245-56.

6. Uchiyama $\mathrm{T}$, Imanishi $\mathrm{K}$, Miyoshi-Akiyama $\mathrm{T}$, Kato $\mathrm{H}$. Staphylococcal superantigens and diseases caused by them. In: AloufJE, PopoffMR: The Comprehensive Sourcebook of Bacterial Protein Toxins, 3rd Edition. New York: Elsevier.2006;830-43.

7. Adalat S, Dawson T, Hackett SJ, Clark JE. In association with the British Paediatric Surveillance Unit. Toxic shock syndrome surveillance in UK children. Arch Dis Child. 2014;99:1078-82.

8. Decleire PY, Blondiaux G, Delaere B, Glupczynski Y. Staphyloccocal scalded skin syndrome in an adult. Acta Clin Belg. 2004;59:365-8.

9. Oono T, Kanzaki H, Yoshioka T, Arata J. Staphylococcal scalded skin syndrome in an adult. Identification of exfoliative toxin $\mathrm{A}$ and $\mathrm{B}$ genes by polymerase chain reaction. Dermatology. 1997;195:268-70.

10. Shirin S, Gottlieb AB, Stahl EB. Staphylococcal scalded skin syndrome in an immunocompetent adult: possible implication of low-dosage prednisone. Cutis. 1998;62:223-4.

11. Centers for Disease Control and Prevention. Toxic shock syndrome (other than Streptococcal) (TSS): 2011 Case Definition. 2014.

12. Chuang YY, Huang YC, Lin TY. Toxic shock syndrome in children: epidemiology, pathogenesis, and management. Paediatr Drugs. 2005; 7:11-25.

13. Takahashi N, Hasegawa H, Komiyama M, et al. Selective excretion of anti-inflammatory cytokine interleukin-10 in a superantigen-inducing neonatal infectious disease. Cytokine. 2009;45:39-43.

14. Takahashi N, Imanishi K, Uchiyama T. Overall picture of an emerging neonatal infectious disease induced by a superantigenic exotoxin mainly produced by methicillinresistant Staphylococcus aureus. Microbiol Immunol. 2013;57:737-45. 